\title{
EDITORIAL
}

\section{Turf wars in radiology: Need for symbiotic relationships}

\author{
Chander Mohan, SM \\ Department of Interventional Radiology, BLK Superspecialty Hospital, New Delhi, India \\ E-mail: brigcmohan@gmail.com
}

Over the past few decades, radiology as a specialty has seen tremendous growth, which has been accompanied by encroachment on imaging modalities by nonradiologists. Various specialists have been competing with radiologists for imaging and interventional procedures related to their specialties, and in few cases have completely taken over the imaging and interventions. These turf wars have led to a renewed sense among the radiology community to protect its own turf of imaging from being taken over by nonradiologists. While monetary gain is definitely one of the major factors behind these wars, there are other sides to the problem as well.

The most glaring example of turf wars is the specialties which need short reaction times such as cardiology, emergency, and critical care medicine. During working hours, the radiology department absorbs most of the imaging burden, however, during nights, the problem becomes grave; the major issue being the availability of the radiologists. Most radiology departments are understaffed during nights because of financial issues, reluctance of staff to night duty scheduling, compensatory offs given later hinder the smooth workflow of the department during the day too. Even if one radiologist is available, he/she is burdened with a load of cross-sectional, emergency, and portable ultrasounds and plain film imaging. As a result, the emergency medicine physicians have started performing and interpreting the ultrasounds with a simple premise that it is better to have a physician with some knowledge than to depend on someone whose availability is uncertain in most cases. Cardiologists have completely taken over the imaging and interventions of their patients. Hence, the boundaries of the turf are becoming ill-defined and interventional radiologists are at a distinct disadvantage due to dependency on referral practice from clinical colleagues.

Another field of strong competition is fetal imaging particularly with ultrasound where the radiologists are competing with obstetricians and gynecologists. In many cases, the obstetricians know what to look for, but may not be expert in how to image it, while radiologists know how to image, but not what exactly to look for in a particular scenario. The radiologists cannot put their foot down on the participation of the obstetricians and gynecologists in the ultrasound as the patients are indeed their patients and without them the entire business would be lost. Furthermore, time constraint is another issue, because for each examination, a radiologist appointment is to be scheduled and the physician then has to wait for the report, due to which many obstetricians and gynecologists prefer to perform routine imaging themselves while reserving the more complex scenarios for the radiologist. Even female patients are more comfortable with this in-house ultrasound particularly for the transvaginal scans. Another issue is the use of defensive reporting in the current medicolegal era where terms such as "cannot exclude" and "clinical correlation" are commonplace in the radiology report, as a result of which the physician is forced to follow up for many conditions, which are much less likely.

Orthopedicians have traditionally been interested in interpreting their own imaging studies. While earlier it was mostly limited to plain films, now the orthopedic physicians want to interpret the magnetic resonance imaging (MRI) themselves. This situation is strikingly similar to neurologists and neurosurgeons who also like to interpret computed tomography (CT) and MRI examinations of their patients. The radiologist's role in many cases is on the verge of being reduced from a "compulsory associate" to more of a "resource person" in difficult cases.

This is an open access article distributed under the terms of the Creative Commons Attribution-NonCommercial-ShareAlike 3.0 License, which allows others to remix, tweak, and build upon the work non-commercially, as long as the author is credited and the new creations are licensed under the identical terms.

\begin{tabular}{|l|l|}
\hline \multicolumn{2}{|c|}{ Access this article online } \\
\hline Quick Response Code: & Website: \\
& www.ijri.org \\
\hline & \\
& \\
\hline
\end{tabular}

Cite this article as: Mohan C. Turf wars in radiology: Need for symbiotic relationships. Indian J Radiol Imaging 2016;26:421-2. 
During the years, interventional pulmonology has also advanced as a specialty and now are competing with the radiologists for performing lung biopsies, bronchial stenting, and pleural drainages. For nodule characterization usually imaging is sufficient, however, the problem starts when a biopsy is required. For peripheral nodules, usually biopsy is performed by a radiologist under CT guidance, while for the nodules near the airway, bronchoscopy can do the job. However, the gray area is the nodules in between the two where both camps consider it a fair game. Similarly, pleural drainage is an area where the two sides compete frequently.

In fact, many radiologists believe that procedures that they have started (e.g., coronary angiography, prostate endorectal and Doppler ultrasound, among others) have been taken away by other specialists, vascular surgeons, general and hepatosurgeons, gynecologists, and urologists, whereas other specialists believe that radiology is invading their territory. Turf wars have been particularly bitter in ultrasound and vascular interventions. The continuing use of diagnostic endoscopic retrograde cholangiopancreatography instead of noninvasive and apparently equally accurate magnetic retrograde cholangiopancreatography is a good example.

As a result, it is necessary that the radiology community has to awaken and equip itself on tactfully dealing with these problems. It is imperative that the number of radiologists should increase to meet the $24 \times 7$ emergency imaging demands of the physicians. This can be dealt to some extent by starting teaching programs with residents and fellows in all the centers dealing with high patient turnover. It has been reported that not only the faculty radiologists but also the residents are better than the emergency physicians in the interpretation of the imaging studies. ${ }^{[1]}$ This has to do with the limited exposure in scanning to most emergency physicians which is easily surpassed by radiology residents. Second is the adoption of teleradiology, particularly in centers with fewer night calls. Even though teleradiology is very helpful for emergency situations, radiologists should desist from using it as a crutch for not being there. It is imperative that we foster the development of a clinical radiologist who is part of the clinical team and cooperates and develops personal contact with the clinical teams even during the wee hours.

The integration of radiologists into multidisciplinary teams involved in patient care such as oncology is also important to review the studies together and decide on the best treatment approach for the patient.
The issue of ignorance of research with the radiology community divided between the academic radiology departments, which are far outnumbered by private practicing radiologists, also needs to be addressed. While the academic departments do most of the research, there is a big pay difference when compared to private practice. That coupled with arranging funding, hiring research staff, and equipment along with a lot of permissions required for conducting research has made research a far from rewarding experience for academic departments who like the private practice groups also face a progressively increasing clinical load. Private practicing radiologists continue to reap the benefits of this by not only knowing the latest advances as well as getting results of long-term studies to test the safety and efficacy of procedures. If we continue to ignore this discrepancy, it will just be a matter of time when even the research in radiology would be taken up by all the specialists we are competing with.

Further, the radiologists need to improve their knowledge of new age modalities such as nuclear medicine and functional imaging and start analyzing the patient in conjunction with the image to increase the relevance of our report. The advancement in research in radiology by the radiologists is needed to accomplish this.

Both the radiologist as well as the physician cannot survive in vacuum. The radiologists of today cannot limit themselves to interpretation of images but have to analyze imaging with due consideration to patient history and pathophysiology and trying to answer the clinical questions in a way that the physicians understand. Tumor boards and clinicoimaging meets should be encouraged and feedback of the physicians carefully analyzed with tailoring of reports to their specialized needs. Often most understandings between the physician and the radiologist can be improved by a quick phone call. Radiologists should increase this communication with their clinical colleagues and also encourage them to ask them for the best imaging test that can be done in a particular scenario. Probably this is the apt time for us to evolve our radiology practice or be prepared to be relegated even in our own specialty.

\section{Reference}

1. Eng J, Mysko WK, Weller GE, Renard R, Gitlin JN, Bluemke DA, et al. Interpretation of Emergency Department radiographs: A comparison of emergency medicine physicians with radiologists, residents with faculty, and film with digital display. Am J Roentgenol 2000;175:1233-8. 